\title{
LUT
}

Lappeenranta

University of Technology

\section{Real-Time monitoring of Laser Scribing Process of CIGS Solar Panels Utilizing High Speed Camera}

Roozbahani Hamid, Marttinen Pekka, Salminen Antti

This is a Final draft

version of a publication

published by IEEE

in IEEE Photonics Technology Letters

DOI: $10.1109 /$ LPT.2018.2867274

Copyright of the original publication: (C) IEEE 2018

Please cite the publication as follows:

Roozbahani H., Marttinen P., Salminen A. (2018). Real-Time monitoring of Laser Scribing Process of CIGS Solar Panels Utilizing High Speed Camera. IEEE Photonics Technology Letters, Vol. 30, Iss. 20, p. 1741-1744. DOI: 10.1109/LPT.2018.2867274 


\title{
Real-Time monitoring of Laser Scribing Process of CIGS Solar Panels Utilizing High Speed Camera
}

\author{
H. Roozbahani, Member, IEEE, P. Marttinen, A. Salminen
}

\begin{abstract}
The aim of this study is to develop a novel method for monitoring of ultra-high speed process of laser scribing with a high-speed camera and evaluate reliability and performance of the developed monitoring system with experimental methods. The case study in this research is monitoring of CIGS solar panels scribing. Due to high-quality requirement of the scribing process of solar panels, it is important to monitor the process in real time to detect possible defects during the process. In order to evaluate the performance of the developed real-time monitoring system, an IPG ytterbium pulsed fiber laser with $20 \mathrm{~W}$ maximum average power is used. A powerful fully programmable industrial computer was chosen for executing real-time image processing and analysis. Algorithms for defect analysis, which are based on Particle Analysis, were developed using LabVIEW ${ }^{\circledR}$ real-time. As a result, the maximum analysis speed of the process was a record breaking of 560 frames per second in real-time including the analysis of each image in real-time. Reliability of the algorithm was evaluated by imaging and analyzing the process with a variable number of defects $2000 \mathrm{~mm} / \mathrm{s}$ and the algorithm detected all defects from the scribing path.
\end{abstract}

Index Terms - Laser scribing, Laser micromachining, Realtime monitoring, CIGS, Solar Panels, High Speed Camera

\section{INTRODUCTION}

Ul Itrafast lasers provide possibilities for many new microprocessing applications because of their capability for thermal ablation and their high peak power. One application is thin film laser scribing, which is used for solar panel manufacturing technology. In laser scribing applications, speed of the process can reach several meters per second, and quality requirements of the process are high. Disturbances in the process can cause defects to the scribing line, which affects the quality of the product. Currently, there is a lack of studies and solutions in process monitoring and quality control. Plenty of papers are published related to the more commonly used laser process applications in high power range such as laser welding [1-5]. However, these processes are significantly slow compared to scribing process, and there is a need to develop a new monitoring system for fast laser scribing.

The aim of this study is to develop a method for monitoring of laser scribing process in real time utilizing a high-speed camera and to detect possible defects in process in real-time. In this case, defect means discontinuance in laser scribing. The speed of the laser scribing process sets high-performance requirements for real-time monitoring. The idea to perform the monitoring is to use a high-speed camera to scan the process and a fully programmable industrial computer to execute fast image processing and defect analysis. Software used for programming and developing the analysis algorithms is LabVIEW.

\section{A. Solar panel manufacturing}

Copper indium gallium selenide (CIGS) is rapidly growing choice as a material for thin-film photovoltaics as a result of its good efficiency. There have been problems to implement laser scribing on CIGS solar modules manufacturing. Therefore, mechanical scribing with a force-controlled stylus has been commonly used manufacturing method. However, the mechanical method has also own problems, example poor edge quality and more importantly, irregularity in scribing lines that demand wide spacing between adjacent scribing lines [6]. As a result, changing scribing method from mechanical scribing to laser scribing, the efficiency of the module is possible to be increased up to four percent [7]. According to Burn et al. (2015)[8] "Specific laser processes selectively remove layers between deposition processes; thereby creating monolithic cell- to-cell interconnections with a very small loss of productive area. Ultrashort pulsed lasers are the tool of choice for scribing $\mathrm{Cu}(\mathrm{In}, \mathrm{Ga}) \mathrm{Se} 2$ (CIGS) thinfilm solar cells as they provide the necessary process selectivity and minimize thermal load on the surrounding material.

\section{B. Monitoring Process}

Utilizing of machine vision applications in laser process monitoring is widely studied [5]. The purpose of the monitoring is gathering information from the process and using that information in developing of quality control methods. During the years, several monitoring methods based on closed-loop algorithms have developed for laser welding process monitoring [2,3]. Advanced digital camera and data processing technology have provided possibility to develop systems based on feature recognition for determining certain features of the process [9]. When the processing parameters are not controlled by real-time monitoring signal, the monitoring system is called an open-loop system. In the open loop system, the process is not adjusted according to the output signal, but the only signal is created which indicates that there is an error in the process. Because quality and reliability of the process is important to be ensured, in recent years the closed loop manufacturing systems have become more popular. In past, only simple sensors, such photodiodes have used in closed loop systems, but recently digital camera technology is improved and they are more commonly used as detectors. Such systems can be used for example for measuring changes in keyhole shape and size or melt pool shape in the laser process and the feedback signal from the camera can be used for controlling process parameters $[1,9]$.

\section{TEST SETUP}

Test setup for experiments including laser, scan head, illumination system, high-speed camera and camera adapter, which are shown in figure 1 . The laser used in the experiments is an IPG ytterbium pulsed fiber laser with $20 \mathrm{~W}$ maximum average power, typical beam quality M2 value of $1.5,1 \mathrm{~mJ}$ max pulse energy, $1.6-1000 \mathrm{kHz}$ pulse repetition rate, and a changeable pulse length from 4 ns to $200 \mathrm{~ns}$ in 8 increments. Scan head optics used in the laser is Scanlab's Hurryscan 14 II with an $\mathrm{f} 100$ tele-centric lens. Working area is $54 \times 54 \mathrm{~mm}^{2}$ and the laser spot size at minimum $28 \mu \mathrm{m}$ but realistically closer to $40 \mu \mathrm{m}$ since it is difficult to keep the laser beam in exact focus with the current setup. 


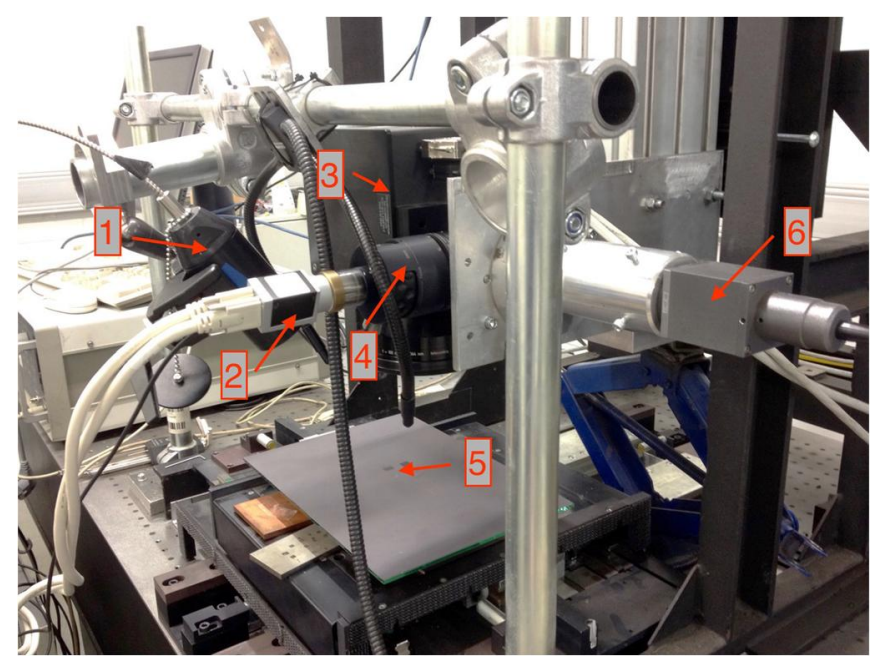

FIG. 1. Test bed for experiments (1. Illumination laser, 2. High-speed camera, 3. Scan head, 4. Camera adapter, 5. Work piece, 6. Pulsed fiber laser).

Used laser control software is SamLight version 3.0.5 build- 0582 by Scaps $\mathrm{GmbH}$. The camera adapter is chosen from Scanlab. It is installed between the scan head and laser flange, allowing the camera to follow laser beam and capturing continuous image from a surface processed by the laser. A dichroic beam splitter inside of the camera adapter passes through the laser beam from the laser source to the working surface, but the back-reflected light from the illuminated surface is decoupled for the camera. The laser beam passes through the beam splitter practically unaffected, the power loss being from 0.5 to 1 percent when the lens is clean. The sharpness of the image is adjusted from the objective unit's focus ring. The focal length of the scanning objective and chip's size of the camera defines the size of the observation field. For an example, focal length of $163 \mathrm{~mm}$ with 2/3-inch censor size, typically produces an image field size of approximately $7.5 \mathrm{~mm} \times 10 \mathrm{~mm}$, maximum optical resolution beings around $10 \mu \mathrm{m}$.

The high-speed camera chosen for the project is Basler acA2000-340km. It is a grey scale Camera Link camera for high-speed imaging in real time. The software of an illumination system controls the camera trigger. The trigger signal is TTL $5 \mathrm{~V}$ and it is delivered from the illumination system to the Camera Link modules D-SUB port where it is delivered forward to the camera via the Camera Link cable. The camera operates as the slave and the illumination software operates as the master. Lighting produced by illumination system is essential for machine vision applications. An illuminated workpiece reflects the light of the lightning source to the camera ship, which collects the light and builds the image for further image processing and analysis [10]. The illumination system used in the laser process monitoring experiments is a high-frequency pulsed diode laser light source Cavilux HF with $500 \mathrm{~W}$ maximum output power. Illumination operates at a wavelength $810 \mathrm{~nm}$. It is used for visualization of high speed and high-temperature processes. High temperatures in laser process produce bright vapor cloud. Without the illumination system, it is impossible to capture accurate images through the brightness. Brightness is filtered out from the image using band-pass filter before sensor of the camera, which passes through only reflected light from illumination laser.

The computation heart of this project is a PXI open PCbased platform created by National Instrument for test, measurement and control systems. The NI PXIe- 8880 is an embedded controller that utilized Intel Xeon CPUs (Central Processing Unit) for fast calculation in different test engineering applications. It is equipped with eight-core processor enabling low latency in calculation. That makes it suitable for high-speed real time image processing applications. The NI PXIe-7966R FPGA module features a DSP-focused Virtex-5 SX95T FPGA and $512 \mathrm{MB}$ of on-board DDR2 DRAM (Dynamic Random Access Memory). This FPGA incorporates 640 Digital Signal Processor (DSP) slices that it can be used to implement digital filters, custom signal processing and fast Fourier transform logic, all of which are commonly found on analog FPGA-based instruments. In addition, the theoretical on board DRAM throughput of 3.2 $\mathrm{GB} / \mathrm{s}$ is necessary when operating on large data sets with the highest-performance adapter modules.

\section{III.EXPERIMENTS}

Image acquisition, processing, and analysis procedure follow the pattern presented in figure 2 .

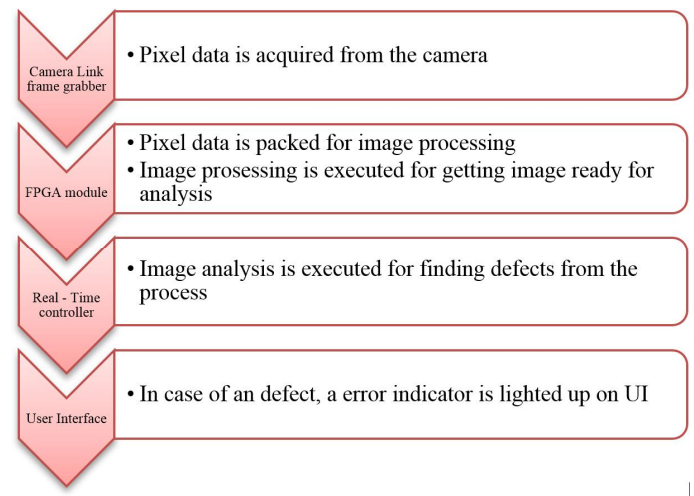

FIG. 2. Image processing and analysis procedure.

For evaluating reliability and performance of the built monitoring system, experiments are performed in four phases. The different phases are shown in figure 3, and they are explained more briefly in following sub sections.

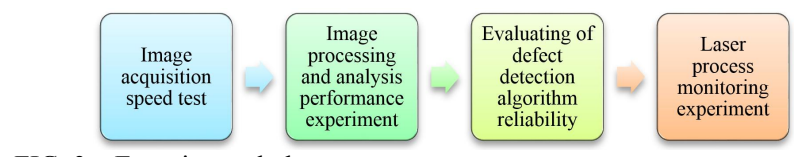

FIG. 3. Experimental phases.

Before the particle analysis can be performed, a grey scale image has to be changed to a binary worm using thresholding operations. There is a corresponding value of the light intensity between 0 and 255 for each pixel, when the lowest light intensity returns value 0 and the highest light intensity returns value 255. By selecting threshold levels within this range, interested information form the image can be highlighted. The thresholding operation returns value 1 for bright pixels and value 0 for dark pixels. Value 1 is shown as a white and value 0 as a black in the images.

When the image is in the binary worm, it is possible to count particles form the image using Particle Analysis algorithms. Before the analysis, a Region Of Interest (ROI) should be chosen from the binary image. In this particular setup, width of the measured observation area is $10 \mathrm{~mm}$ and length of the scribing line in frame is $4.7 \mathrm{~mm}$. The length of the scribing line is about half of the observation area because laser spot is middle of the area. The ROI is shown in figure 4, which is defined manually from the image using pixel calculator. The particle analysis is executed only inside of that area. Principle of the analysis is based on the number of the particles. 


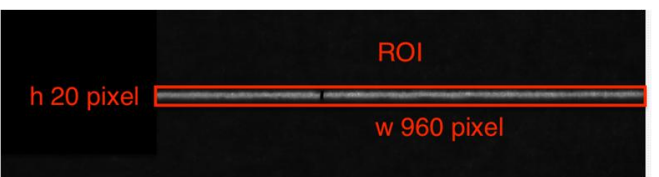

FIG. 4. Selection of the ROI.

When the scribing line is coherent, there is only one particle inside of the ROI and if there is discontinuous (defect) in scribing, the ROI includes at least two particles. Impurity on the surface can create small particles into the ROI. These small particles can distort the result of the defect analysis. Therefore, the particles have to be removed before the analysis. These operations can be performed using an advanced morphology operation in LabVIEW. Figure 5 shows the image after the threshold operation. Small-unwanted particles inside the ROI could be seen in the figure (one of them are zoomed). Unwanted particles should be removed from the image before the particle analysis. The minimum particle size is set to $3 \times 3$ pixel, and all smaller particles are removed. Figure 6 shows a binary image without unwanted particles.

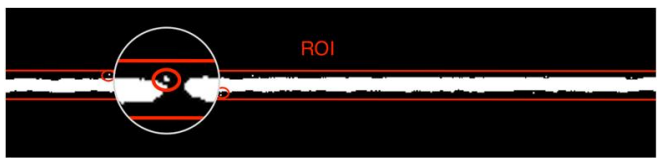

FIG. 5. Threshold image with unwanted particles.

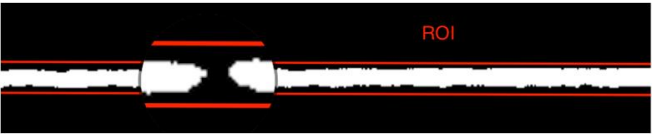

FIG. 6. Threshold image where unwanted particles are removed.

As it can be seen from figure 7, after the morphology operations, there are two particles inside of the ROI. All unwanted particles are removed, and the image is ready for the particle analysis. As a result, the analysis returns number of the particles and in this particular case, the defect can be detected because the image includes two particles instead of one.

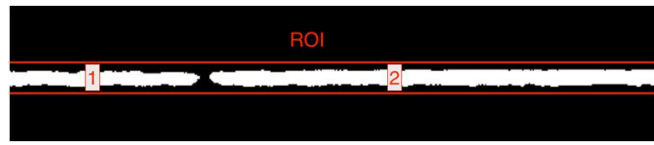

FIG. 7. Image ready for particle analysis (1. Particle number 1, 2. Particle number 2).

Performance of the defect detection can be defined by imaging surface where is an example one particle. By increasing triggering frequency step-by-step, analysis algorithms analyses more images during a second. After all CPU power is used, and maximum analysis speed is achieved, acquired images starting to fill the buffer of the FIFOs. The phenomena can be noticed from the user interface. Maximum analysis speed of laser scribing can be calculated multiplying maximum defect detection speed (fps) with the length of scribing line fitted in one frame.

Evaluation of reliability of the defect detection algorithms can be performed by analyzing images from a scribing line. Three $20 \mathrm{~mm}$ long lines are scribed to the $1 \mathrm{~mm}$ thick aluminum piece, which has $15 \mu \mathrm{m}$ thick anodized coating.

The programmed scribing path is shown in figure 8 . As it can be seen from the figure, one line is programmed without discontinuity, another with one $0.1 \mathrm{~mm}$ long discontinuity and third with two $0.1 \mathrm{~mm}$ long discontinuities. The programmed discontinuities on the lines are presenting defects in cribbing. The scribed lines on the coated aluminum are show in figure
9. The real length of the discontinuities is little less than programmed, the measured length being $80 \mu \mathrm{m}$.

\section{$\sqrt{4}$}

FIG. 8. CAD drawings in SAMLight laser control software.

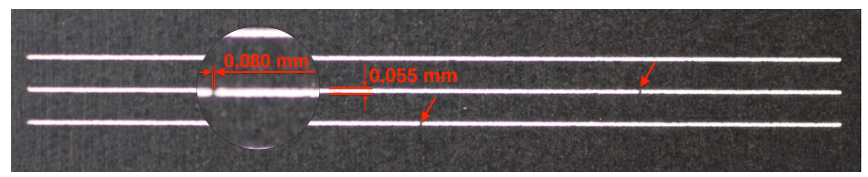

FIG. 9. Scribed lines for reliability experiments.

After the scribing process, the laser scanner is programmed to follow the same scribing path and the camera is set to imaging scribing line through the scan head. After imaging and analyzing the whole path, a number of defects detected during the analysis should be same than a number of defects programmed to the path. Because the laser is turned off during the experiment, active laser illumination is not needed and it was replaced with four white LED lights for easier directing of the lightning. The LED lights were directed to the surface of the test piece from all directions (left, right, front and back) and as straight from up as it was possible when the smallest possible angle being about 25 degrees. The distance of the LED lights from the surface was about $9 \mathrm{~cm}$. Required frame rate for the defect analysis can be calculated by dividing the laser processing speed by length of the scribing line in the ROI. In this case, the processing speed is set to $2000 \mathrm{~mm} / \mathrm{s}$ and length of the measured scribing line is $4.7 \mathrm{~mm}$. According to these values, needed frame rate for the analysis is $426 \mathrm{fps}$. For achieving small overlap in imaging, the speed is set to $430 \mathrm{fps}$.

Laser monitoring experiment are performed using same scribing path than in the previous experiment where reliability of defect detection algorithm was tested. The difference is that now the experiment is performed during the laser scribing process. Active laser illumination is used for lightning and the filter is set to the camera adaptor in front of the camera for filtering out all other light except lighting from the illumination laser, including reflected light from the pulsed laser. Illumination laser is directed as straight to perpendicular to the surface as it is possible, when the angle being about 45 degrees. Physical dimensions of the illumination laser lens and the scan head set the limit for the angel.

\section{RESULTS}

As it can be seen in table 1 , the maximum frame rate of the camera with resolution $960 \times 20$ is $10900 \mathrm{fps}$. After that, the speed did not increase even if the camera was triggered faster. During the experiment, the FIFOs on the RT was not able to read the data as quickly than the data was written into the FIFOs on the FPGA causing data overflows in the RT's FIFOs. Maximum image acquisition speed without data overflows was $2700 \mathrm{fps}$ when the analysis algorithms and the displays were disconnected. Maximum defect detection speed was tested with the defect analysis algorithm using $1500 \mu \mathrm{s}$ exposure time and non-moving image. As a result, the limit for the analysis speed was $560 \mathrm{fps}$. With $560 \mathrm{fps}$, it is possible to monitor laser-scribing process up to $2.7 \mathrm{~m} / \mathrm{s}$ in real-time, which is a high speed in comparison to current used scribing speeds in the industry. When the speed exceeded that limit, the buffers of the FIFOs on RT started to fill and analysis was not performed in real time anymore. The reliability experiment was performed executing defect analysis for tree different scribing line. Camera frame rate was set to $430 \mathrm{fps}$, allowing 
one percent overlap in imaging and exposure time was set to $70 \mu \mathrm{s}$. Threshold range [10,255] provided best results and it is used in the experiment. The results are presented in table 2.

TABLE I

RESULTS OF THE IMAGE ACQUISITION AND DEFECT ANALYSIS PERFORMANCE EXPERIMENTS.

\begin{tabular}{l|c}
\hline \hline Maximum camera frame rate & $10900 \mathrm{fps}$ \\
\hline Maximum Image acquisition speed - FPGA to RT & $2700 \mathrm{fps}$ \\
\hline Maximum defect analysis speed & $560 \mathrm{fps}$ \\
\hline \hline
\end{tabular}

TABLE II

RESULTS OF THE DEFECT DETECTION ALGORITHM RELIABILITY EXPERIMENT.

\begin{tabular}{l|c|c|c}
\hline \hline Number of defects in scribing & 0 & 1 & 2 \\
\hline Algorithm reliability experiment & $10 / 10$ & $10 / 10$ & $10 / 10$ \\
\hline \hline
\end{tabular}

As it is illustrated in table 2, the defect detection algorithm has detected all the defects successfully. In the scribing monitoring experiment, the defect analysis did work due difficulties with the illumination provided by active laser illumination. Images captured from the scribing line with the laser illumination are shown below. The image before image processing is shown in figure 10 and the threshold image is shown in figure 11 .

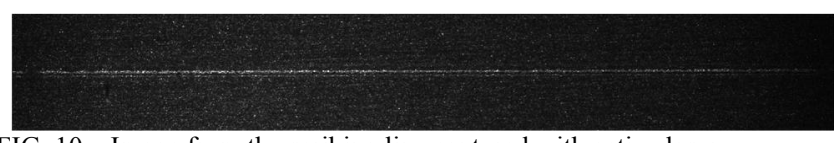

FIG. 10. Image from the scribing line captured with active laser illumination.

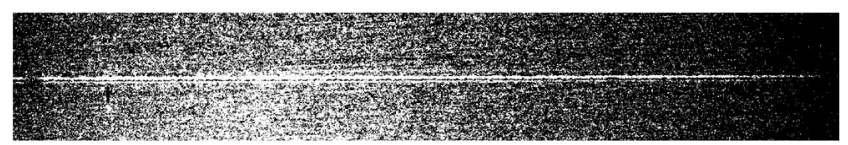

FIG. 11. Threshold image from scribing line captured with active laser illumination.

\section{CONCLUSION AND SUMMARY}

The purpose of the study was finding a method for monitoring of laser scribing process with a high-speed camera in real time and evaluating performance and reliability of the method. The study was divided into a theoretical and an experimental part.

In the theoretical part, first, some study was carried out to get knowledge from a digital camera and machine vision technology. The knowledge was used for choosing the proper camera and for setting the camera parameters for fast imaging. In addition, some study was carried out for helping in development of image processing and analysis algorithms. It was also important to improve knowledge in laser scribing technology and study existed laser-monitoring applications. It was concluded that existed monitoring systems are typically implemented to monitor laser processes such a laser welding, where the speed of the process is sufficient low. The speed of the existed monitoring applications is not fast enough to implement real-time monitoring in laser scribing applications. That means the new monitoring system was needed to be developed which can detect defects during the fast laser processes. According to the study in theoretical part, there could be market potential for fast real time laser scribing process monitoring application, an example in solar cell technology, where benefits of the laser scribing are studied.

In the experimental part of the study, the test setup was developed for fast real-time monitoring. Chosen high-speed camera for the imaging was Basler acA2000-340km, which is Camera Link camera with CMOS sensor. The camera provides ten taps parallel pixel readout from the sensor and flexible ROI selection. High performance PXI-system was built to execute image processing and defect analysis.

The choice of image analysis algorithm for the defect analysis is based on particle analysis due its simplicity. Simple algorithm can analyze images faster than complicated algorithm such pattern recognition.

Purpose of the experiments is evaluating performance and reliability of the monitoring system. Preferring to the results, the maximum defect detection speed is $560 \mathrm{fps}$, when the defect analysis sets the limit for the maximum speed. This speed represents a monitor speed up to to $2.7 \mathrm{~m} / \mathrm{s}$ in real-time. It is possible to increase the speed by adding additional parallel computation power (FPGA units) to the current real-time computer. The reliability of the defect detection was evaluated with two experiments. The first experiment was performed imaging the scribing line when the laser was turned off. The laser scanner was programmed to follow in advance scribed line $2000 \mathrm{~mm} / \mathrm{s}$, while the defect detection was set to execute analysis $430 \mathrm{fps}$. Illumination was implemented using white light source from multiple directions. The experiment was successful, and defect analysis algorithm did not miss any defects during the experiment. The second experiment was performed during the laser process. The experiment was similar than the first one, but now laser was turned on. The purpose was to monitor the process and find the defects during the laser process. The results were not as good as in first experiment without laser. Because laser was on, the white light illumination had to be replaced with powerful active laser illumination. However, directing of the laser illumination was demanding and it was hard to get enough contrast between scribing line and surrounding surface. Performing defect analysis was impossible due poor illumination. However, according to the results of experiments it can be concluded that error detection algorithms work with proper illumination and method for real-time laser scribing monitoring is found.

\section{VI.REFERENCES}

[1] Tenner, F., Brock, C., Klämpfl, F. \& Schmidt, M. 2015. "Analysis of the correlation between plasma plume and keyhole behavior in laser metal welding for the modeling of the keyhole geometry." Optics and Lasers in Engineering, Vol. 64.pp. 32-41.r, 2015, p. 8)

[2] Fennander, H., Kyrki, V., Fellman, A., Salminen, A. \& Kälviäinen, H. 2009. "Visual measurement and tracking in laser hybrid welding." Machine Vision and Applications, Vol. 20, Iss. 2. pp. 103-118.

[3] Huang, W. \& Kovacevic, R. 2012. "Development of a real-time laser-based machine vision system to monitor and control welding processes." The International Journal of Advanced Manufacturing Technology, Vol. 63, Iss. 1. pp $\underline{235-248 .}$.

[4] Chen, H., Lv, F., Lin, T. \& Chen, S. 2009. "Closed-Loop Control of Robotic Arc Welding System with Full-penetration Monitoring”, vol. 56. pp. 565-578.

[5] Fidali, M. \& Jamrozik, W. 2013. "Diagnostic method of welding process based on fused infrared and vision images." Infrared Physics \& Technology, Vol. 61.pp. 241-253.

[6] Rekow, M., Murison, R., Panarello, T., Dunsky, C., Dinkel, C., Nikumb, S., Pern, J. \& Mansfield, L. 2010. "CIGS P1, P2, P3 Scribing Processes using a Pulse Programmable Industrial Fiber Laser."

[7] Eberhardt, G., Banse, H., Wagner, U. \& Peschel, T. 2010. "Structuring of thin film solar cells, Proc.'

[8] Burn, A., Heger, C., Buecheler, S., Nishiwaki, S., Tiwari, A., Ziltener, R., Bremaud, D., Krainer, L., Spuehler, G. \& Romano, V. 2015. "All laser scribing for monolithic interconnection of $\mathrm{Cu}(\mathrm{In}, \mathrm{Ga})$ Se thin film solar cells Optimization, validation, and assessment for industrial production "

[9] Purtonen, T., Kalliosaari, A. \& Salminen, A. 2014. "Monitoring and Adaptive Control of Laser Processes. Physics Procedia"; 8th International Conference on Laser Assisted Net Shape Engineering LANE 2014, Vol. 56. pp. 1218-1231.

[10] Liu, Z., Ukida, H., Niel, K. \& Ramuhalli, P. 2015. "Industrial Inspection with Open Eyes: Advance with Machine Vision Technology. Integrated Imaging and Vision Techniques for Industrial Inspection." Part of the series Advances in Computer Vision and Pattern Recognition. pp. 1-37. 